\title{
Molecular Mechanism Underlying the Plant NRT1.1 Dual-Affinity Nitrate Transporter
}

\author{
Ji Sun ${ }^{1 * t}$ and Ning Zheng ${ }^{1,2 *}$ \\ ${ }^{1}$ Department of Pharmacology, University of Washington, Seattle, WA, USA, ${ }^{2}$ Howard Hughes Medical Institute, University of \\ Washington, Seattle, WA, USA
}

\section{OPEN ACCESS}

Edited by:

Mario L. Diaz,

Universidad de La Laguna, Spain

Reviewed by:

Gabriel Krouk,

Centre National de la Recherche

Scientifique, France

Eiji Nambara,

University of Toronto, Canada Maria Isabel Bahamonde Santos,

Pompeu Fabra University, Spain

${ }^{*}$ Correspondence: Ji Sun

jsun@mail.rockefeller.edu.

Ning Zheng

nzheng@u.washington.edu

${ }^{\dagger}$ Present Address:

Ji Sun,

Laboratory of Molecular Neurobiology and Biophysics, The Rockefeller

University, New York, NY, USA

Specialty section:

This article was submitted to Membrane Physiology and Membrane

Biophysics,

a section of the journa

Frontiers in Physiology

Received: 23 October 2015 Accepted: 30 November 2015 Published: 18 December 2015

Citation:

Sun J and Zheng N (2015) Molecular Mechanism Underlying the Plant

NRT1.1 Dual-Affinity Nitrate Transporter. Front. Physiol. 6:386. doi: 10.3389/fphys.2015.00386
Nitrate $\left(\mathrm{NO}_{3}^{-}\right)$is one of the most important sources of mineral nitrogen, which also serves as a key signaling molecule for plant growth and development. To cope with nitrate fluctuation in soil that varies by up to four orders of magnitude, plants have evolved high- and low-affinity nitrate transporter systems, consisting of distinct families of transporters. Interestingly, the first cloned nitrate transporter in Arabidopsis, NRT1.1 functions as a dual-affinity transporter, which can change its affinity for nitrate in response to substrate availability. Phosphorylation of a threonine residue, Thr101, switches NRT1.1 from low- to high-affinity state. Recent structural studies have unveiled that the unmodified NRT1.1 transporter works as homodimers with Thr101 located in close proximity to the dimer interface. Modification on the Thr101 residue is shown to not only decouple the dimer configuration, but also increase structural flexibility, thereby, altering the substrate affinity of NRT1.1. The structure of NRT1.1 helps establish a novel paradigm in which protein oligomerzation and posttranslational modification can synergistically expand the functional capacity of the major facilitator superfamily (MFS) transporters.

Keywords: NRT1.1, dimer, dual-affinity, nitrate transporter, major facilitator superfamily, transceptor

\section{INTRODUCTION}

Nitrate $\left(\mathrm{NO}_{3}^{-}\right)$is critical for plants, both as a primary nutrient and as an important signaling molecule (Crawford, 1995; Krouk et al., 2010a). Nitrogen (N) is a key constituent of nucleotides and amino acids, thus essential for life. In plants, about $2-5 \%$ of the dry biomass is made up of nitrogen, which is largely acquired by plant roots in the form of nitrate (Xu et al., 2012). Nitrate also functions as a critical signaling ion and regulates many aspects of plant growth and development (Castaings et al., 2011; Bouguyon et al., 2012), including nitrate-related gene expression (Wang et al., 2003), root architecture (Forde, 2014), seed dormancy (Alboresi et al., 2005), and flowering time (Castro Marín et al., 2011).

Active nitrate uptake through membrane transporters by plant roots represents the key first step of nitrogen acquisition (Dechorgnat et al., 2011). As sessile organisms, plants have evolved sophisticated nitrate transporter systems in response to the fluctuating nitrate environments (Wang et al., 2012). In Arabidopsis thaliana, one of the most well studied nitrate transporters is NRT1.1 (CHL1 or NPF6.3), which is a multifunctional protein with a crucial role in both nitrate acquisition and signaling. Firstly, NRT1.1 is a dual-affinity transporter, which can facilitate nitrate assimilation over a wide range of nitrate concentrations (Liu et al., 1999). Secondly, NRT1.1 has recently been shown to serve as a nitrate sensor, regulating the gene expression of other nitrate transporters such as NRT2.1 (Krouk et al., 2006; Ho et al., 2009). Last by not least, NRT1.1 also contributes 
to the nitrate-regulated auxin translocation besides nitrate transport and sensing (Krouk et al., 2010b). In this review, we will mainly focus on the transporter functions of NRT1.1 and summarize recent structure-function studies, which have shed light on the molecular mechanism underlying its dual-affinity activity.

\section{Nitrate Transporters in Arabidopsis}

To cope with the fluctuation of nitrate level, plants have evolved two complementary nitrate transporter systems with distinct kinetics properties (Forde, 2000; Nacry et al., 2013; Krapp et al., 2014). The low-affinity transporter system (LATS), which consists members of the NRT1/PTR family (recently renamed as NPF family) (Steiner et al., 1995; Léran et al., 2014), drives nitrate uptake at millimolar concentration. This family shares sequence homology to SLC15/PTR/PepT/POT family of peptide transporter family in animal. In plants, the NRT1/PTR family has functionally diverged with individual members recognizing different substrates including peptides (Tsay et al., 2007), plant hormones (Krouk et al., 2010b), glucosinolates (Nour-Eldin et al., 2012), and nitrate (Tsay et al., 1993). The high-affinity transporter system (HATS), comprising the NRT2/NNP family, facilitates nitrate uptake with Michaelis constant $\left(\mathrm{K}_{m}\right)$ value in the micromolar range. Studies suggest that some NRT2 members require a second gene product for their functional activity. In the case of NRT2.1, NAR2 is needed to mediate its nitrate uptake (Kotur et al., 2012).

NRT1/PTR and NRT2/NNP together constitute a large NRT transporter family in plants, which are proton-coupled symporters and belong to the major facilitator superfamily (MFS) (Pao et al., 1998). Together there are 53 NRT1 genes and 7 NRT2 genes in Arabidopsis (Tsay et al., 2007). Besides ensuring the root capacity of nitrate uptake, NRT transporters are also involved in subsequent loading and unloading of nitrate to and from the xylem vessels, allowing its distribution to aerial organs or its remobilization from old leaves (Chiu et al., 2004; Lin et al., 2008; Fan et al., 2009; Wang and Tsay, 2011).

\section{NRT1.1 is a Dual-Affinity Transporter}

The Arabidopsis NRT1.1 (CHL1 or NPF6.3) protein is the founding member of the NRT1/PTR family. It was firstly cloned as a gene product that is responsible for chlorate sensitivity in Arabidopsis (Tsay et al., 1993). The NRT1.1 protein contains 12 membrane-spanning segments and confers proton-coupled nitrate transport activity.

Interestingly, NRT1.1 is essential for both high- and lowaffinity nitrate absorptions in Arabidopsis. It presents a biphasic uptake curve in response to environmental nitrate availability, and thus functions as a dual-affinity transporter (Huang et al., 1996; Wang et al., 1998; Liu et al., 1999; Liu and Tsay, 2003). NRT1.1 shares sequence homology with members of the NRT1/PTR family that constitutes the LATS, and was initially shown to be a low-affinity nitrate transporter. Later, it was found that plants with nrt1.1 mutation were also defective in highaffinity nitrate uptake, suggesting that NRT1.1 might be a dualaffinity transporter. Further experiments in plants and oocytes demonstrate that the uptake curve of NRT1.1 is biphasic with a
$\mathrm{Km}$ of $\sim 50 \mu \mathrm{M}$ for high-affinity phase of uptake and $\sim 4 \mathrm{mM}$ for the low-affinity phase (Liu and Tsay, 2003).

The mode of action of the dual-affinity function of NRT1.1 is regulated through phosphorylation modification on a key threonine residue, Thr101 (Liu and Tsay, 2003). Thr101 is located at the intracellular side between the third and fourth transmembrane helix (TM) of NRT1.1. When phosphorylated by the CIPK23 kinase (Ho et al., 2009), NRT1.1 functions as a high-affinity nitrate transporter. When unphosphorylated, it works as a low-affinity transporter. Furthermore, mutations of Thr101 preventing or mimicking phosphorylation can effectively convert the dual-affinity transporter into a monophasic lowaffinity or high-affinity transporter, respectively. This regulatory mechanism of NRT1.1 allows for the rapid adaption to changing nitrate levels.

\section{Molecular Basis of the Dual-Affinity Function}

How does Thr101 phosphorylation switch the transporter affinity of NRT1.1? Recently, two independent studies revealed the crystal structures of unmodified NRT1.1, shedding light on the molecular basis of the working mechanism of this transporter (Parker and Newstead, 2014; Sun et al., 2014). In both studies, NRT1.1 was crystallized with two molecules in each asymmetric unit (ASU), despite the different polypeptide boundaries, crystallization conditions, and space groups (Figure 1A). The two molecules in the ASU are almost identical to each other and adopt the canonical MFS fold, which is characterized by 12 transmembrane helices (TMs) with a central linker connecting the amino-terminal (TM1-TM6) and carboxyl-terminal (TM7TM12) helix bundles (Law et al., 2008; Yan, 2013). The transporter is captured in the inward conformation with the substrate-binding site accessible from the cytosol side.

Both in vitro biochemical studies and cell-base fluorescence resonance energy transfer (FRET) assays suggest that the NRT1.1 dimer could be physiologically relevant (Sun et al., 2014). First of all, NRT1.1 dimer is observed in two different crystal forms. The two adjacent non-crystallographic related NRT1.1 molecules are in the same orientation with their amino-terminal halves facing and interacting with each other. This overall topology is perfectly compatible with its transporter function in the cell membrane (Figure 1A). Secondly, the dimer interface, burying a surface area of more than $2160 \AA^{2}$, has high shape-complementarity that is considered as an important determinant of helix packing specificity in membrane or micelles (Fleming et al., 1997; Mackenzie and Fleming, 2008; Chen et al., 2009). Thirdly, in vitro crosslinking experiments show that transient dimer of NRT1.1 could form in detergent solutions. Consistent with these in vitro observations, cell-based FRET assay also demonstrates that NRT1.1 oligomerizes in the membrane of Xenopus oocytes, where the dual-affinity function of NRT1.1 can be recapitulated.

More importantly, the oligomerization state of NRT1.1 could be affected by the phosphorylation modification on NRT1.1 (Sun et al., 2014). In the crystal structure, the key residue, Thr101 is buried in a hydrophobic pocket that is located right next to the dimer interface (Figure 1A). Phosphorylation of Thr101 was predicted to introduce electrostatic and conformational changes 

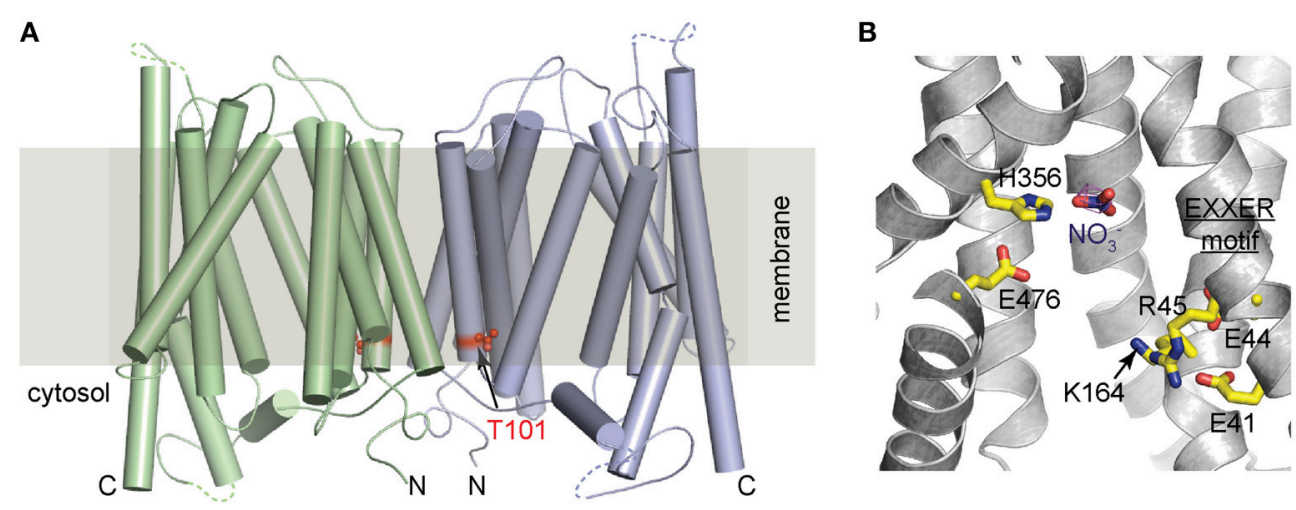

FIGURE 1 | Crystal structure of NRT1.1. (A) A cylinder representation of the NRT1.1 dimer with Thr101 shown as red spheres. The two monomers are colored in pale green and light blue. Within each monomer, the amino- and carboxyl-terminus are indicated by $\mathrm{N}$ and $\mathrm{C}$. (B) Key residues involved in substrate binding and proton coupling. Nitrate and the side chain of the neighboring histidine, His356, EXXER motif, Glu476, and Lys164 are shown as sticks.

in its vicinity and disrupt the dimeric configuration of NRT1.1. This prediction was confirmed by oocyte-base FRET assays. Both wild-type NRT1.1 and the phosphorylation defective mutant NRT1.1-T101A generate robust and comparable FRET signals. In contrast, the phosphomimetic mutation, NRT1.1-T101D failed to show any significant signal, indicating a spatial separation of the two monomers. These data suggest a phosphorylationdependent dimerization switching mechanism for the dualaffinity transporter: unmodified NRT1.1 forms structurally coupled dimers and works as a low-affinity transporter, whereas phosphorylated NRT1.1 undergoes dimer decoupling and adopts a high-affinity state.

Besides decoupling the dimer, phosphorylation on Thr101 also alters the transporter properties of each monomer (Parker and Newstead, 2014). NRT1.1-T101D has a lower melting temperature, indicating increased structure flexibility. Meanwhile, the transport rate of the phosphomimetic variant is also higher than wild-type protein by 2.8 -folds in the liposomebase uptake assay. Therefore, enhanced structure flexibility of NRT1.1 through Thr101 phosphorylation leads to an increased transporter rate, which results in a lower $\mathrm{K}_{\mathrm{m}}$.

Taken together, phosphorylation on the Thr101 toggles NRT1.1 between the high- and low-affinity states by decoupling the dimer and increasing structure flexibility (Tsay, 2014; Figure 2). When nitrate is abundant, NRT1.1 is dephosphorylated. At this state, the transporter adopts a dimeric conformation, has lower structural flexibility, and functions as a low-affinity transporter. When the environmental nitrate concentration drops, NRT1.1 becomes phosphorylated on the Thr101 residue at the dimer interface, which decouples the NRT1.1 dimer. As a result, the phosphorylated transporter gains increased structural flexibility and uptake activity, and works as a high-affinity transporter.

\section{Substrate Recognition and Proton Coupling in NRT1.1}

A key question regarding to the working mechanism of a transporter is how substrate is recognized. High-resolution structures of bacterial nitrate transporter and nitrate/nitrite exchanger (NarU and NarK) from NNP family had been documented before the crystal structures of NRT1.1 were determined (Yan et al., 2013; Zheng et al., 2013). Nitrate in these two bacterial transporters is coordinated by two opposing conserved arginine residues through ionic interactions, which provides high-affinity contacts between the transporters and nitrate. Distinct from NarU and NarK, the overall substratebinding pocket of NRT1.1 is mostly hydrophobic, with the exception of one histidine residue, His356 on TM7 (Figure 1B), which is shown to be critical in nitrate recognition. Despite the resolution limit, discernable electron density of nitrate is located in close vicinity to His356, mutation of which eliminated substrate uptake both at high and low nitrate concentrations. Notably, His356 is not conserved in transporters of the NRT1/PTR family, suggesting that other NRT1/PTR members may have different substrate recognition mechanism. The unique chargeable histidine residue at the substrate binding pocket provides a plausible explanation for the high-affinity activity acquired by NRT1.1. Sequence differences in the substrate binding sites may also explain why NRT/PTRs are able to recognize substrates as diverse as nitrate, peptides and plant hormones.

In addition to His356, an EXXER motif and a potential salt bridge between Lys164 and E476 (Figure 1B) are shown to be critical in proton coupling by uptake assays in liposomes and oocytes (Parker and Newstead, 2014; Sun et al., 2014). The importance of these residues is also validated by two independent assays, two-electrode voltage clamp (TEVC) and a novel fluorescent method, in which the activity of NRT1.1 was monitored through its tight correlation with FRET readout of fluorescent proteins linked to $\mathrm{N}$ - and C-terminus of the transporter (Ho and Frommer, 2014).

\section{MFS oligomerization}

Besides NRT1.1, numerous MFS transporters including TetL (Safferling et al., 2003), GalP (Zheng et al., 2010), GLUT1 (Graybill et al., 2006), hRFC (Hou and Matherly, 2009), 


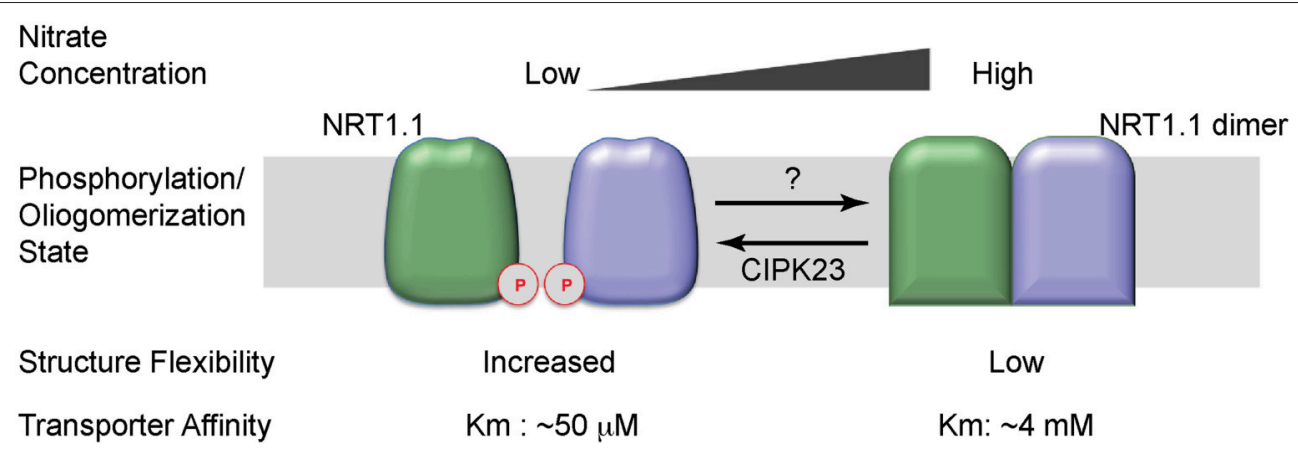

FIGURE 2 | Working model of the dual-affinity transporter, NRT1.1. When nitrate concentration is low, NRT1.1 is phosphorylated (P) at the amino-acid residue Thr101. The phosphorylation modification disrupts the dimer configuration and increases the structural flexibility of NRT1.1, leading to a high affinity for nitrate. When nitrate is abundant, Thr101 is dephosphorylated. NRT1.1 works as a coupled dimer, which has decreased structural flexibility, rendering the transporter in a low-affinity state. The two NRT1.1 molecules are colored in pale green and light blue. The phosphorylation modification is indicated by "P" in a red cycle.

MCT8 (Fischer et al., 2015), and LacS (Veenhoff et al., 2001) have been shown to exist as homo-oligomers. Functional importance of the MFS oligomerization has been indicated in several studies. For example, the lactose transporter LacS from Streptococcus Thermophiles functions as cooperative dimers with two substrate translocation pathways, and co-reconstitution of functional and defective transporters disrupts proton-coupled lactose uptake, suggesting a dominant-negative effect (Veenhoff et al., 2001). The human monocarboxylate transporter 8 (MCT8), mutation of which underlies the cause of a severe $\mathrm{X}$ linked psychomotor retardation (known as the Allan-HerndonDudley syndrome), forms functional homodimers in vivo. Native occurring pathogenic mutations from patients that abolish the transporter function also largely affect the formation of homodimers, suggesting the potential significance of the transporter oligomerization (Fischer et al., 2015).

In the case of NRT1.1, phosphorylation-controlled dimerization provides a novel aspect on how dynamic transporter oligomerization involves in functional modulation. The dimerization state of NRT1.1 is fine-tuned by the posttranslational modification. Upon phosphorylation and dephosphorylation of the Thr101 residue, the transporter oligomerization state is regulated, which in turn further changes the structure flexibility of NRT1.1 and switches the transporter between high- and low-affinity modes. Thus, structure-function studies of NRT1.1 not only establishes a structural framework for understanding the dual-affinity activity, but also reveals how posttranslational modification and protein oligomerization can synergistically expand the functional capacity of an MFS transporter.

\section{Perspective}

In this review, we focused on the recent progress on the molecular basis of the dual-affinity activity of the key nitrate transporter, NRT1.1. Through structural and biochemical studies, the oligomerization state and the structural flexibility of the transporter are proposed to play a key role in the phosphorylation dependent transporter affinity switch. Yet many important questions remain to be answered to further our understanding on this important molecule. For example, what is the structure of the phosphorylated transporter like? Crystallographic analysis of the phosphorylated form will help us visualize the conformational difference between the high- and low-affinity states, and obtain a better picture of the working mechanism. Since the high-affinity property of NRT1.1 has been challenged (Glass and Kotur, 2013), it will be also valuable to systematically characterize the function of NRT1.1 at different oligomerization and phosphorylation states in vitro. By mutating and disrupting the dimer interface (Robertson et al., 2010), a phosphorylationindependent NRT1.1 monomer could be potentially obtained. Structural and functional analyses of such a mutant could help address the questions as to whether phosphorylation alters the properties of the transporter beyond controlling its oligomerization state and whether unphosphorylated NRT1.1 monomer is functional. Furthermore, how Thr101 becomes modified by the CIPK23 kinase if this residue is buried in a hydrophobic pocket at the dimer interface? It is proposed that the phosphorylation might occur when the transporter is at the occluded or outward conformation, yet this hypothesis awaits more evidence. Last but not least, do both protomer copies of the NRT1.1 dimer have to be phosphorylated before the affinity state of the transporter is changed? Designing a functional NRT1.1 concatemer with two individually manipulable copies of the transporter might help shed light on this and other questions.

Another interesting aspect of the NRT1.1 protein is its nitrate sensor function, which exemplifies the transceptor (transporter with sensor function) paradigm in Arabidopsis (Ho et al., 2009; Gojon et al., 2011). Transceptors have been found in many organisms from E. coli to mammals (Thevelein and Voordeckers, 2009), and almost all transceptors are important in nutrients transport and sensing (Donaton et al., 2003; Schwöppe et al., 2003; Hyde et al., 2007; Rebsamen et al., 2015; Wang et al., 2015). Recently, the mammalian amino acid transporter, SLC38A9 was identified as an amino acid sensor for lysosome-based activation of mTORC1, indicating the importance of transceptor in human disease (Abraham, 2015). A fundamental question 
underlying the working mechanism of a transceptor is how substrate translocation and sensing is coupled? In the case of NRT1.1, its transceptor function also exhibits a biphasic manner switched by Thr101 phosphorylation, as the phosphorylation and non-phosphorylated forms of NRT1.1 have distinct signaling properties (Bouguyon et al., 2015). It will be interesting to know whether NRT1.1 dimerization is also involved in the sensor affinity switch. An equally important question is how nitrate signal is transduced through protein-protein interaction. In the crystal structure of NRT1.1, the well-ordered N-terminal loop at the dimer interface on the intracellular side presents a conserved docking interface that may have a putative role in nitrate signaling by recruiting downstream signal molecules such as kinases and phosphatases. Further genetic and biochemical studies will be needed to support this hypothesis.

Last but not least, NRT1.1 facilitates nitrate-regulated auxin transport. It has been reported that NRT1.1 could use both nitrate and auxin as its substrates, and enable soil nitrate availability to regulate the lateral root development (Krouk

\section{REFERENCES}

Abraham, R. T. (2015). Cell biology. Making sense of amino acid sensing. Science 347, 128-129. doi: 10.1126/science.aaa4570

Alboresi, A., Gestin, C., Leydecker, M. T., Bedu, M., Meyer, C., and Truong, H. N. (2005). Nitrate, a signal relieving seed dormancy in Arabidopsis. Plant Cell Environ. 28, 500-512. doi: 10.1111/j.1365-3040.2005.01292.x

Bouguyon, E., Brun, F., Meynard, D., Kubeš, M., Pervent, M., Leran, S., et al. (2015). Multiple mechanisms of nitrate sensing by Arabidopsis nitrate transceptor NRT1.1. Nat. Plants 1:15015. doi: 10.1038/nplants.2015.15

Bouguyon, E., Gojon, A., and Nacry, P. (2012). Nitrate sensing and signaling in plants. Semin. Cell Dev. Biol. 23, 648-654. doi: 10.1016/j.semcdb.2012.01.004

Castaings, L., Marchive, C., Meyer, C., and Krapp, A. (2011). Nitrogen signalling in Arabidopsis: how to obtain insights into a complex signalling network. J. Exp. Bot. 62, 1391-1397. doi: 10.1093/jxb/erq375

Castro Marín, I., Loef, I., Bartetzko, L., Searle, I., Coupland, G., Stitt, M., et al. (2011). Nitrate regulates floral induction in Arabidopsis, acting independently of light, gibberellin and autonomous pathways. Planta 233, 539-552. doi: 10.1007/s00425-010-1316-5

Chen, L., Merzlyakov, M., Cohen, T., Shai, Y., and Hristova, K. (2009). Energetics of ErbB1 transmembrane domain dimerization in lipid bilayers. Biophys. J. 96, 4622-4630. doi: 10.1016/j.bpj.2009.03.004

Chiu, C. C., Lin, C. S., Hsia, A. P., Su, R. C., Lin, H. L., and Tsay, Y. F. (2004). Mutation of a nitrate transporter, AtNRT1:4, results in a reduced petiole nitrate content and altered leaf development. Plant Cell Physiol. 45, 1139-1148. doi: $10.1093 / \mathrm{pcp} / \mathrm{pch} 143$

Crawford, N. M. (1995). Nitrate: nutrient and signal for plant growth. Plant Cell 7, 859-868. doi: 10.1105/tpc.7.7.859

Dechorgnat, J., Nguyen, C. T., Armengaud, P., Jossier, M., Diatloff, E., Filleur, S., et al. (2011). From the soil to the seeds: the long journey of nitrate in plants. J. Exp. Bot. 62, 1349-1359. doi: 10.1093/jxb/erq409

Donaton, M. C., Holsbeeks, I., Lagatie, O., Van Zeebroeck, G., Crauwels, M., Winderickx, J., et al. (2003). The Gap1 general amino acid permease acts as an amino acid sensor for activation of protein kinase A targets in the yeast Saccharomyces cerevisiae. Mol. Microbiol. 50, 911-929. doi: 10.1046/j.13652958.2003.03732.x

Fan, S. C., Lin, C. S., Hsu, P. K., Lin, S. H., and Tsay, Y. F. (2009). The Arabidopsis nitrate transporter NRT1.7, expressed in phloem, is responsible for source-to-sink remobilization of nitrate. Plant Cell 21, 2750-2761. doi: 10.1105/tpc.109.067603

Fischer, J., Kleinau, G., Müller, A., Kühnen, P., Zwanziger, D., Kinne, A., et al. (2015). Modulation of monocarboxylate transporter 8 oligomerization by et al., 2010b). This reveals a surprising mechanism by which plants adjust their root architecture for soil exploitation, and raises another interesting question as to how NRT1.1 recognizes two structurally different substrates. Further studies will be required to fully uncover the role of NRT1.1 in nitrate transport and signaling, which enable plants to adapt to the fluctuated nitrate environment. Ultimately, these studies will potentially facilitate the development of new technologies for increasing crop yields as well as reducing nitrogen pollution in modern agriculture.

\section{AUTHOR CONTRIBUTIONS}

Both authors contributed to the drafting and revising the work.

\section{FUNDING}

This work is supported by the Howard Hughes Medical Institute and the National Science Foundation (NSF MCB-1157561). specific pathogenic mutations. J. Mol. Endocrinol. 54, 39-50. doi: 10.1530/JME14-0272

Fleming, K. G., Ackerman, A. L., and Engelman, D. M. (1997). The effect of point mutations on the free energy of transmembrane alpha-helix dimerization. J. Mol. Biol. 272, 266-275. doi: 10.1006/jmbi.1997.1236

Forde, B. G. (2000). Nitrate transporters in plants: structure, function and regulation. Biochim. Biophys. Acta 1465, 219-235. doi: 10.1016/S00052736(00)00140-1

Forde, B. G. (2014). Nitrogen signalling pathways shaping root system architecture: an update. Curr. Opin. Plant Biol. 21, 30-36. doi: 10.1016/j.pbi.2014.06.004

Glass, A. D., and Kotur, Z. (2013). A re-evaluation of the role of Arabidopsis NRT1.1 in high-affinity nitrate transport. Plant Physiol. 163, 1103-1106. doi: 10.1104/pp.113.229161

Gojon, A., Krouk, G., Perrine-Walker, F., and Laugier, E. (2011). Nitrate transceptor(s) in plants. J. Exp. Bot. 62, 2299-2308. doi: 10.1093/jxb/erq419

Graybill, C., Van Hoek, A. N., Desai, D., Carruthers, A. M., and Carruthers, A. (2006). Ultrastructure of human erythrocyte GLUT1. Biochemistry 45, 8096-8107. doi: 10.1021/bi060398x

Ho, C. H., and Frommer, W. B. (2014). Fluorescent sensors for activity and regulation of the nitrate transceptor CHL1/NRT1.1 and oligopeptide transporters. Elife 3:e01917. doi: 10.7554/eLife.01917

Ho, C. H., Lin, S. H., Hu, H. C., and Tsay, Y. F. (2009). CHL1 functions as a nitrate sensor in plants. Cell 138, 1184-1194. doi: 10.1016/j.cell.2009.07.004

Hou, Z., and Matherly, L. H. (2009). Oligomeric structure of the human reduced folate carrier: identification of homo-oligomers and dominant-negative effects on carrier expression and function. J. Biol. Chem. 284, 3285-3293. doi: 10.1074/jbc.M807206200

Huang, N. C., Chiang, C. S., Crawford, N. M., and Tsay, Y. F. (1996). CHL1 encodes a component of the low-affinity nitrate uptake system in Arabidopsis and shows cell type-specific expression in roots. Plant Cell 8, 2183-2191. doi: 10.1105/tpc.8.12.2183

Hyde, R., Cwiklinski, E. L., Macaulay, K., Taylor, P. M., and Hundal, H. S. (2007). Distinct sensor pathways in the hierarchical control of SNAT2, a putative amino acid transceptor, by amino acid availability. J. Biol. Chem. 282, 19788-19798. doi: 10.1074/jbc.M611520200

Kotur, Z., Mackenzie, N., Ramesh, S., Tyerman, S. D., Kaiser, B. N., and Glass, A. D. (2012). Nitrate transport capacity of the Arabidopsis thaliana NRT2 family members and their interactions with AtNAR2.1. New Phytol. 194, 724-731. doi: 10.1111/j.1469-8137.2012.04094.x

Krapp, A., David, L. C., Chardin, C., Girin, T., Marmagne, A., Leprince, A. S., et al. (2014). Nitrate transport and signalling in Arabidopsis. J. Exp. Bot. 65, 789-798. doi: $10.1093 / \mathrm{jxb} / \mathrm{eru001}$ 
Krouk, G., Crawford, N. M., Coruzzi, G. M., and Tsay, Y. F. (2010a). Nitrate signaling: adaptation to fluctuating environments. Curr. Opin. Plant Biol. 13, 266-273. doi: 10.1016/j.pbi.2009.12.003

Krouk, G., Lacombe, B., Bielach, A., Perrine-Walker, F., Malinska, K., Mounier, E., et al. (2010b). Nitrate-regulated auxin transport by NRT1.1 defines a mechanism for nutrient sensing in plants. Dev. Cell 18, 927-937. doi: 10.1016/j.devcel.2010.05.008

Krouk, G., Tillard, P., and Gojon, A. (2006). Regulation of the high-affinity NO3uptake system by NRT1.1-mediated NO3- demand signaling in Arabidopsis. Plant Physiol. 142, 1075-1086. doi: 10.1104/pp.106.087510

Law, C. J., Maloney, P. C., and Wang, D. N. (2008). Ins and outs of major facilitator superfamily antiporters. Annu. Rev. Microbiol. 62, 289-305. doi: 10.1146/annurev.micro.61.080706.093329

Léran, S., Varala, K., Boyer, J. C., Chiurazzi, M., Crawford, N., Daniel-Vedele, F., et al. (2014). A unified nomenclature of NITRATE TRANSPORTER 1/PEPTIDE TRANSPORTER family members in plants. Trends Plant Sci. 19, 5-9. doi: 10.1016/j.tplants.2013.08.008

Lin, S. H., Kuo, H. F., Canivenc, G., Lin, C. S., Lepetit, M., Hsu, P. K., et al. (2008). Mutation of the Arabidopsis NRT1.5 nitrate transporter causes defective root-to-shoot nitrate transport. Plant Cell 20, 2514-2528. doi: 10.1105/tpc.108.060244

Liu, K. H., Huang, C. Y., and Tsay, Y. F. (1999). CHL1 is a dual-affinity nitrate transporter of Arabidopsis involved in multiple phases of nitrate uptake. Plant Cell 11, 865-874. doi: 10.1105/tpc.11.5.865

Liu, K. H., and Tsay, Y. F. (2003). Switching between the two action modes of the dual-affinity nitrate transporter CHL1 by phosphorylation. EMBO J. 22, 1005-1013. doi: 10.1093/emboj/cdg118

Mackenzie, K. R., and Fleming, K. G. (2008). Association energetics of membrane spanning alpha-helices. Curr. Opin. Struct. Biol. 18, 412-419. doi: 10.1016/j.sbi.2008.04.007

Nacry, P., Bouguyon, E., and Gojon, A. (2013). Nitrogen acquisition by roots: physiological and developmental mechanisms ensuring plant adaptation to a fluctuating resource. Plant Soil 370, 29. doi: 10.1007/s11104-013-1645-9

Nour-Eldin, H. H., Andersen, T. G., Burow, M., Madsen, S. R., Jørgensen, M. E., Olsen, C. E., et al. (2012). NRT/PTR transporters are essential for translocation of glucosinolate defence compounds to seeds. Nature 488, 531-534. doi: 10.1038/nature11285

Pao, S. S., Paulsen, I. T., and Saier, M. H. Jr. (1998). Major facilitator superfamily. Microbiol. Mol. Biol. Rev. 62, 1-34.

Parker, J. L., and Newstead, S. (2014). Molecular basis of nitrate uptake by the plant nitrate transporter NRT1.1. Nature 507, 68-72. doi: 10.1038/nature13116

Rebsamen, M., Pochini, L., Stasyk, T., De Araújo, M. E., Galluccio, M., Kandasamy, R. K., et al. (2015). SLC38A9 is a component of the lysosomal amino acid sensing machinery that controls mTORC1. Nature 519, 477-481. doi: 10.1038 /nature 14107

Robertson, J. L., Kolmakova-Partensky, L., and Miller, C. (2010). Design, function and structure of a monomeric ClC transporter. Nature 468, 844-847. doi: 10.1038 /nature09556

Safferling, M., Griffith, H., Jin, J., Sharp, J., De Jesus, M., Ng, C., et al. (2003). TetL tetracycline efflux protein from Bacillus subtilis is a dimer in the membrane and in detergent solution. Biochemistry 42, 13969-13976. doi: 10.1021/bi035173q

Schwöppe, C., Winkler, H. H., and Neuhaus, H. E. (2003). Connection of transport and sensing by UhpC, the sensor for external glucose-6-phosphate in Escherichia coli. Eur. J. Biochem. 270, 1450-1457. doi: 10.1046/j.14321033.2003.03507.x

Steiner, H. Y., Naider, F., and Becker, J. M. (1995). The PTR family: a new group of peptide transporters. Mol. Microbiol. 16, 825-834. doi: 10.1111/j.13652958.1995.tb02310.x
Sun, J., Bankston, J. R., Payandeh, J., Hinds, T. R., Zagotta, W. N., and Zheng, N. (2014). Crystal structure of the plant dual-affinity nitrate transporter NRT1.1. Nature 507, 73-77. doi: 10.1038/nature13074

Thevelein, J. M., and Voordeckers, K. (2009). Functioning and evolutionary significance of nutrient transceptors. Mol. Biol. Evol. 26, 2407-2414. doi: $10.1093 / \mathrm{molbev} / \mathrm{msp} 168$

Tsay, Y. F. (2014). Plant science: how to switch affinity. Nature 507, 44-45. doi: 10.1038 /nature 13063

Tsay, Y. F., Chiu, C. C., Tsai, C. B., Ho, C. H., and Hsu, P. K. (2007). Nitrate transporters and peptide transporters. FEBS Lett. 581, 2290-2300. doi: 10.1016/j.febslet.2007.04.047

Tsay, Y. F., Schroeder, J. I., Feldmann, K. A., and Crawford, N. M. (1993). The herbicide sensitivity gene CHL1 of Arabidopsis encodes a nitrate-inducible nitrate transporter. Cell 72, 705-713. doi: 10.1016/0092-8674(93)90399-B

Veenhoff, L. M., Heuberger, E. H., and Poolman, B. (2001). The lactose transport protein is a cooperative dimer with two sugar translocation pathways. EMBO J. 20, 3056-3062. doi: 10.1093/emboj/20.12.3056

Wang, R., Liu, D., and Crawford, N. M. (1998). The Arabidopsis CHL1 protein plays a major role in high-affinity nitrate uptake. Proc. Natl. Acad. Sci. U.S.A. 95, 15134-15139. doi: 10.1073/pnas.95.25.15134

Wang, R., Okamoto, M., Xing, X., and Crawford, N. M. (2003). Microarray analysis of the nitrate response in Arabidopsis roots and shoots reveals over 1,000 rapidly responding genes and new linkages to glucose, trehalose-6phosphate, iron, and sulfate metabolism. Plant Physiol. 132, 556-567. doi: 10.1104/pp.103.021253

Wang, S., Tsun, Z. Y., Wolfson, R. L., Shen, K., Wyant, G. A., Plovanich, M. E., et al. (2015). Metabolism. Lysosomal amino acid transporter SLC38A9 signals arginine sufficiency to mTORC1. Science 347, 188-194. doi: 10.1126/science. 1257132

Wang, Y. Y., Hsu, P. K., and Tsay, Y. F. (2012). Uptake, allocation and signaling of nitrate. Trends Plant Sci. 17, 458-467. doi: 10.1016/j.tplants.2012.04.006

Wang, Y. Y., and Tsay, Y. F. (2011). Arabidopsis nitrate transporter NRT1.9 is important in phloem nitrate transport. Plant Cell 23, 1945-1957. doi: $10.1105 /$ tpc. 111.083618

Xu, G., Fan, X., and Miller, A. J. (2012). Plant nitrogen assimilation and use efficiency. Annu. Rev. Plant Biol. 63, 153-182. doi: 10.1146/annurev-arplant042811-105532

Yan, H., Huang, W., Yan, C., Gong, X., Jiang, S., Zhao, Y., et al. (2013). Structure and mechanism of a nitrate transporter. Cell Rep. 3, 716-723. doi: 10.1016/j.celrep.2013.03.007

Yan, N. (2013). Structural advances for the major facilitator superfamily (MFS) transporters. Trends Biochem. Sci. 38, 151-159. doi: 10.1016/j.tibs.2013.01.003

Zheng, H., Taraska, J., Merz, A. J., and Gonen, T. (2010). The prototypical $\mathrm{H}+$ /galactose symporter GalP assembles into functional trimers. J. Mol. Biol. 396, 593-601. doi: 10.1016/j.jmb.2009.12.010

Zheng, H., Wisedchaisri, G., and Gonen, T. (2013). Crystal structure of a nitrate/nitrite exchanger. Nature 497, 647-651. doi: 10.1038/nature12139

Conflict of Interest Statement: The authors declare that the research was conducted in the absence of any commercial or financial relationships that could be construed as a potential conflict of interest.

Copyright $(\odot) 2015$ Sun and Zheng. This is an open-access article distributed under the terms of the Creative Commons Attribution License (CC BY). The use, distribution or reproduction in other forums is permitted, provided the original author (s) or licensor are credited and that the original publication in this journal is cited, in accordance with accepted academic practice. No use, distribution or reproduction is permitted which does not comply with these terms. 